\title{
REDUNDANT IMAGE REPRESENTATION VIA MULTI-SCALE DIGITAL RADON PROJECTIONS
}

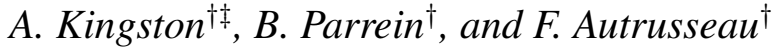 \\ † IRCCyN-IVC, Ecole polytechnique de l'Université de Nantes, \\ rue Christain Pauc, La Chantrerie, 44306 Nantes Cedex 3, FRANCE. \\ $\ddagger$ Department of Applied Mathemathics, RSPhySE, \\ The Australian National University, Canberra ACT 0200, AUS.
}

\begin{abstract}
A novel ordering of digital Radon projections co-efficients is presented here that enables progressive image reconstruction from low resolution to full resolution. The digital Radon transform applied here is the Mojette transform first defined by Guedon et al. in [1]. The Mojette transform is a natural way to generate redundancy to any specified degree and has been demonstrated to be useful for redundant representation for robust data storage and transmission. Combining this with the wavelet transform facilitates compression, i.e., joint source-channel coding, along with the additional property of scalability.
\end{abstract}

Index Terms - Radon Transforms, image representation, image coding, image communication, distibuted source coding

\section{INTRODUCTION}

Scalable image representation is very powerful in communication. It enables the cohabitation of different displays with a range of screen resolutions, progressive image transmission refinement from low to full resolution, efficient browsing of image databases, and establishes a hierarchy that prepares the image for compression. However image coding streams are non resilient over noisy channels. A natural way to overcome this is to introduce some form of redundancy to protect the streams from losses. This work presents a scheme to combine the power of scalability with the robustness of redundancy using a digital Radon transform.

The Radon transform (RT) is an invertible mapping from a continuous 2D function to a set of $1 \mathrm{D}$ continuous projections at all angles $\theta \in[0, \pi)$. A projection at angle, $\theta$, is obtained as the linear integration of the function over all parallel lines with gradient $\tan \theta$. The RT is utilised in areas ranging from

The majority of this work was conducted while AK held a postdoctoral position at IUniversite de Nantes. This work is partially supported by the MILES project, funded by the Région Pays de la Loire, France medical tomography (CT, MRI, ultrasound) to astronomy and seismology.

A digital Radon transform (DRT) is an ideal way to achieve distribution as the image is spread over many projections. The Finite Radon Transform (FRT) [2] is a well known, mathematically elegent, digitisation of the RT that preserves the major RT properties. It is restricted to $p \times p$ arrays, (where $p$ is prime), mapped to a torus. However, by definition FRT is not a redundant transform. A particularly useful DRT for this purpose is the Mojette transform since the number of projections and degree of redundancy is completely tunable.

The Mojette transform is very similar to the FRT but removes the periodic boundary conditions. It is an entirely discrete, exactly invertible mapping between an image and projections which requires only the addition operation. Like the FRT, it retains the major properties of the RT, however, it also introduces the property of redundancy. It was first proposed by Guédon et al in 1995 [1] in the context of psychovisual image coding. It has since been applied in many aspects of image processing such as image analysis, image watermarking, image encryption, and tomographic reconstruction from projections. The unique properties of the transform have also made it a useful channel coding tool with applications in robust data transmission and distributed data storage. A summary of the evolution and applications of the Mojette transform to date can be found in [3].

Mojette projections are a natural way to achieve redundant distibution of images (or any data). This paper presents a method to obtain multi-scale projections which can achieve joint source-channel coding AND add the power of scalablitly. Firstly, the Mojette transform is outlined in section 2. The method to achieve redundant representation is then described in section 3, and demonstrated with a small example. Finally the concept of a multi-scale representation of the projections is presented in section 4 , again with a small example. Some concluding remarks and the future directions of this research are given in section 5 . 


\section{THE MOJETTE TRANSFORM}

\subsection{Mojette projection}

The Mojette transform is an exact, discrete form of the classical Radon transform defined for specific "rational" projection angles. Like the RT, the Mojette transform represents the image as a set of projections, however in contrast, the Mojette transform has an exact inverse from a finite number of discrete projections (as few as 1 depending on the angle set). The rational projection angles, $\theta_{i}$, are defined by a set of vectors $\left(p_{i}, q_{i}\right)$ as $\theta_{i}=\tan ^{-1}\left(q_{i} / p_{i}\right)$, as depicted in Fig. 1a for $\left(p_{i}, q_{i}\right)=(2,1)$. These vectors must respect the condition that $p_{i}$ and $q_{i}$ are coprime (i.e., $\operatorname{gcd}\left(p_{i}, q_{i}\right)=1$ ) and, since tan is $\pi$-periodic, $q_{i}$ is restricted to be positive except for the case $\left(p_{i}, q_{i}\right)=(1,0)$. The transform domain of an image is a set of projections where each element or "bin", $b$, corresponds to the sum of the pixels centred on the line of projection, $b=l p_{i}-k q_{i}$, as depicted in Fig. 1a. This is a linear transform defined for each projection angle by the operator:

$$
\operatorname{proj}_{p_{i}, q_{i}}(b)=\sum_{k=-\infty}^{\infty} \sum_{l=-\infty}^{\infty} f(k, l) \Delta\left(b+k q_{i}-l p_{i}\right)
$$

where $(k, l)$ defines the position of an image pixel and $\Delta(b)$ is the Kronecker delta function which is 1 when $b=0$ and zero otherwise. Invertible projections can be obtained not only with addition but using any linear operation; Other practically useful operations include modulo 256 addition and bitwise XOR. The Mojette transform, $\mathcal{M}_{I} f(k, l)$, corresponds to the set of $I$ projections as $\mathcal{M}_{I} f(k, l)=\left\{\operatorname{proj}_{p_{i}, q_{i}}, i \in[1 \ldots I]\right\}$.

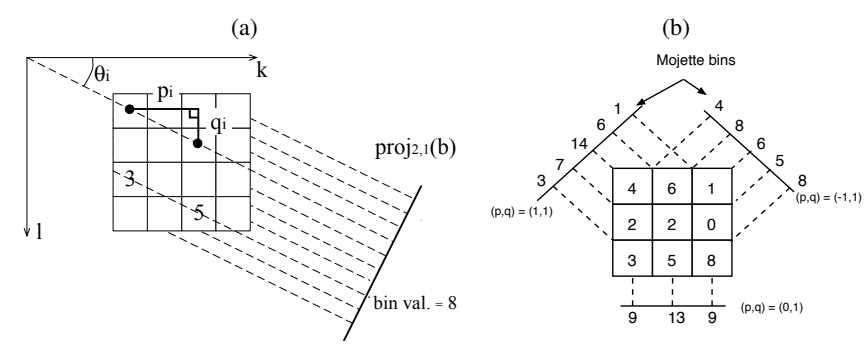

Fig. 1. (a) A depiction of $\left(p_{i}, q_{i}\right), \theta_{i}$, and the method of projection. (b) An invertible Mojette transform of a $3 \times 3$ example image using direction vectors $\{(1,0),(1,1),(-1,1)\}$

The principle difference from the classical Radon transform is this variable sampling rate on each projection, which depends on the chosen angle as $\sqrt{p_{i}^{2}+q_{i}^{2}}$. This can be seen for the different projections in Fig. 1b which demonstrates the Mojette transform for the directions set $S=$ $\{(1,0)(-1,1)$ and $(1,1)\}$. The number of bins, $B_{i}$, for each projection depends on the chosen direction vector $\left(p_{i}, q_{i}\right)$, and for a $P \times Q$ image is found as

$$
B_{i}=(Q-1)\left|p_{i}\right|+(P-1) q_{i}+1
$$

The algorithmic complexity of the Mojette transform for a $P \times Q$ image with $I$ projections is $O(P Q I)$.

\subsection{Criteria for reconstructibility}

Since the set of projection directions is selected arbitrarily, a criterion is required to determine if a set of projections is sufficient to uniquely reconstruct the data. Katz [4] developed a test for data with rectangular support in a very similar context. He showed that if the following criterion is satisfied, any $P \times Q$ dataset can be uniquely reconstructed:

$$
P \leq \sum_{i=1}^{I}\left|p_{i}\right| \quad \text { or } \quad Q \leq \sum_{i=1}^{I} q_{i}
$$

This result has been extended in an independent manner by Normand and Guédon [5] to apply to data with compact support of any shape.

\subsection{Reconstruction from projections}

The standard inverse Mojette transform is a fast and simple algorithm [5]. Searching for and updating 1-1 pixel-bin correspondence enables a simple iterative procedure to recover the image. The bin value is back-projected into the pixel and subtracted from the corresponding bins in all other projections. The number of pixels belonging to the corresponding bins is also decremented. The algorithmic complexity of the inverse Mojette transform for a $P \times Q$ image with $I$ projections is $\mathrm{O}(P Q I)$ [5]. Figure 2 shows one possibility of the first three steps of the inverse Mojette transform of the example projections given in Fig. 1.

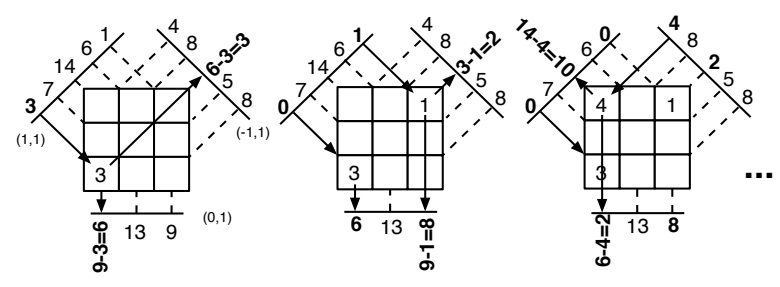

Fig. 2. Three first possible steps of the inverse Mojette transform of the projections obtained in Fig. 1.

This "accounting" inverse has been improved by Normand et al in [6] where the need to search for 1-1 pixel-bin correspondence was replaced by a structured reconstruction sequence that can be quickly calculated for any set of projection angles. A slow but stable algebraic solution using the conjugate gradient method can be used for the case of noisy projections [7]; The search for a preconditioner continues. 


\section{REDUNDANT IMAGE REPRESENTATION}

Redundancy is necessary in communication since channels are noisy and introduce errors. When a message is not correctly received, it is repeated or can be recovered if sent in a form which enables correction. Most channel coding techniques utilise Forward Error Correcting (FEC) codes where the error rate of the channel is predicted from statistics and a rate of redundancy is adjoined to the message for possible error detection and correction at the receiver. The range of redundancy allocation is very large, it can in principle be infinite as for rateless codes, (e.g., Luby transform code [8]), where codewords continue to be sent until the receiver signals that decoding is complete. For the case of Joint Source Channel (JSC) coding [9], the rate must be tunable in order to satisfy both the source priorities and channel statistics. Linear algebra is commonly used in this framework but rarely discrete geometry, (as for the Mojette transform), which provides simple linear complexity and deterministic decoding.

Since projections sets can be selected arbitrarily for the Mojette transform, it is a natural mechanism to redundantly represent data. Simply utilise a projection set with more projections than that required by Katz criterion (3). The number of extra projections is determined by the degree of redundancy required. Then if some projections are corrupted or lost, in either storage or transmission, the image can still be reconstructed from the remaining projections. Each projection contains the entire image and thus have the same sum; This property can be used for error detection.

For example, take a $48 \times 48$ pixel image which requires a redundancy of $33 \%$. If it is projected with four direction vectors: $( \pm 15,2),( \pm 21,2)$. Then Katz criterion is certainly satisfied $\left(\sum_{i}\left|p_{i}\right|=72 \geq 48\right)$ and in fact it is satisfied by taking any 3 of the 4 projections. So storing these projections on 4 separate nodes, or transmitting them over 4 separate channels, implies any one of these can fail and the image can still be recovered.

Adding redundacy does of course increase the amount of information, so introducing some form of compression to the projections is desirable. The following section describes one method to achieve this and also incorporate multi-resolutional capabilities by applying the Wavelet transform.

\section{MULTI-SCALE REDUNDANT IMAGE REPRESENTATION}

The Discrete Wavelet Transform (DWT) is comprised of two operators, a scaling operator $\Phi$, and a wavelet operator $\Psi$, which can both be reduced to a convolution followed by a downsampling, $\mathcal{D}$. Let $\mathcal{W}$ denote either (and in some cases both) of these operators in the following.

A property of the Radon transform known as the convolution property also applies to the Mojette transform; The 2D convolution of images can be performed as a set of 1D convolutions over the images projections. Provided the Mojette direction vectors, $\left(p_{i}, q_{i}\right)$, are consistent with downsampling, the Mojette transform is compatible with the DWT. That is the Mojette projection of the DWT applied to the image, i.e., $\mathcal{M}(\mathcal{W}(f))$, is equivalent to the Mojette projection of the DWT applied to the Mojette projection of the image, i.e., $\mathcal{M}(\mathcal{W})(\mathcal{M}(f))$.

What does it mean to have Mojette projection direction vectors that are consistent with the downsampling? Essentially it is required that the pixels intersected by a common line after downsampling are the only pixels this line intersects in the original image. For a downsampling $\left(s_{k}, s_{l}\right)$, where the image is downsampled by $s_{k}$ in the $k$-direction and by $s_{l}$ in the $l$-direction, this occurs when the Mojette direction vector, $(p, q)$, can be formed with $\left(s_{k}, s_{l}\right)$ as one Hadamard product, i.e., $\operatorname{gcd}\left(s_{k}, p\right)=s_{k} \wedge \operatorname{gcd}\left(s_{l}, q\right)=s_{l}$. For example the direction vector $(15,2)$ can be downsampled by $(1,2),(3,1)$, or $(3,2)$. A downsampling by $(3,2)$ has been depicted in Fig. 3 , note that the direction vector $(15,2)$, which can be written as the Hadamard product $(3,2) \bullet(5,1)$, becomes $(5,1)$ in the downsampled image.

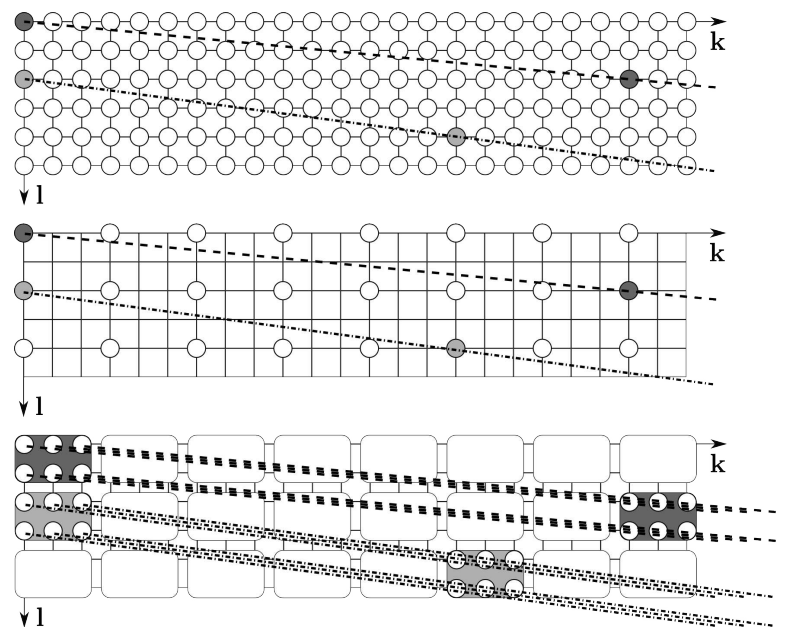

Fig. 3. Lines with the direction vectors $(15,2)$ [dot-dash] and $(21,2)[$ dash] in the original image are consistent with a downsampling of $(3,2)$.

Return to the example from the previous section, a $48 \times$ 48 image with four projections. Note that the image and all projections can be downsampled by $(3,2)$ to result in a $16 \times 24$ image with projection direction vectors $( \pm 5,1)$, and $( \pm 7,1)$. Therefore we can apply $\mathcal{M}(\mathcal{W})$ with this downsampling to the projections and apply some entropy coding to the result to achieve compression. This example has been depicted in Fig. 4. Here the 1D projections have been presented as 2D images of width $p_{i}$, this is possible since Mojette projections retain the 2D image auto-correlation.

These projections maintain the redundant representation as any 1 of these projections can be lost without losing re- 


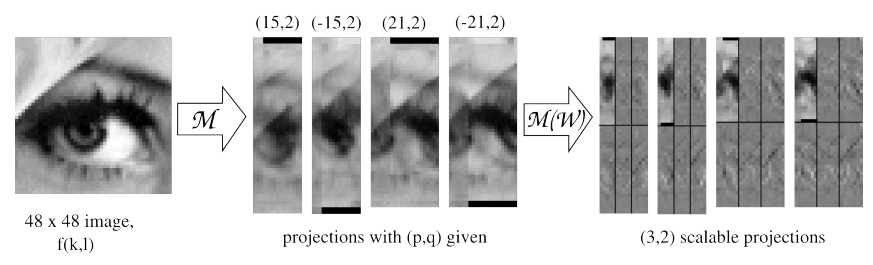

Fig. 4. Take the four projections with direction vectors $( \pm 15,2)$, and $( \pm 21,2)$ and apply $\mathcal{M}(\mathcal{W})$ (Haar like in this case) with a downsampling of $(3,2)$.

constructibility, they are compressed, and the image can be reconstructed from the projections at a low resolution and then a higher resolution. Taking 3 of the four projections, there are two reconstruction schemes as depicted in Fig. 5. The first is simply to apply $\mathcal{M}(\mathcal{W})^{-1}$ to the projections and then apply the inverse Mojette, $\mathcal{M}^{-1}$. The second, more useful, path is to take the low resolution components of each projection, $\mathcal{M}(f)_{L}$, and reconstruct a low resolution image, $\mathcal{M}^{-1}\left(\mathcal{M}(f)_{L}\right)$, and upsample, $\mathcal{D}^{-1}$, then to reconstruct the detail image, $\mathcal{M}^{-1}\left(\mathcal{M}(f)_{H}\right)$, and combine to reconstruct the full resolution image.

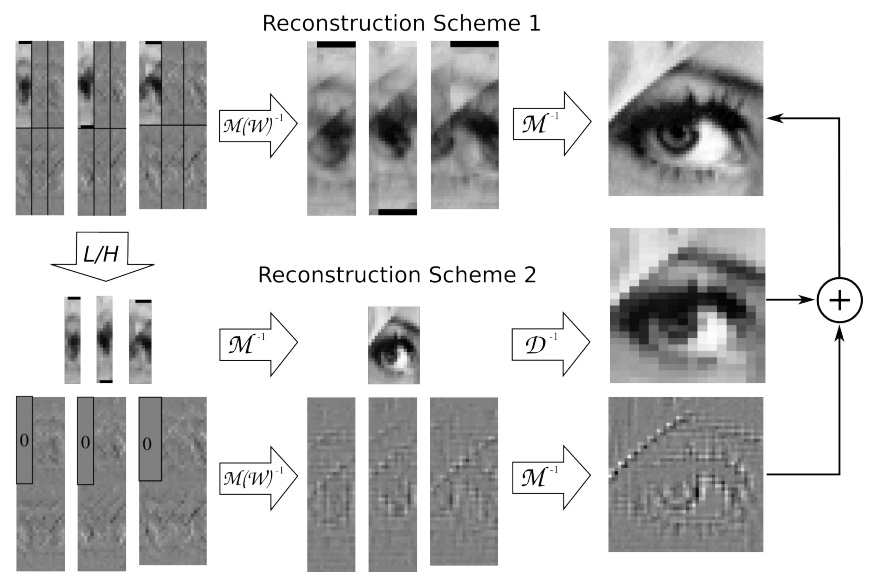

Fig. 5. The two possible reconstruction paths for multi-scale mojette projections.

\section{CONCLUSIONS AND FUTURE WORK}

This preliminary study has presented a novel technique to achieve a scalable image representation with redundancy. It is based on a digital version of the Radon transform, known as the Mojette transform, which has very useful distribution properties with a tunable redundancy.

The RT convolution property also applies to Mojette projections. This implies that the discrete Wavelet transform is compatible with Mojette projections provided the downsampling is consistent with the projection direction vectors. An example demonstrating the scalable projections was given. The multi-scale nature enables progressive image reconstruction from low to full resolution.

We are currently developing the theory of this technique to enable dyadic downsampling and attempting to use the multi-scale approach to speed up algebraic reconstruction by the conjugate gradient method which is very robust to noise. This could then enable lossy compression.

\section{REFERENCES}

[1] JP. Guédon, D. Barba, and N. Burger, "Psychovisual image coding via an exact discrete Radon transform," in Proc. Visual Communications \& Image Processing (VCIP), Lance T. Wu, Ed., Taipei, Taiwan, May 1995, pp. 562-572.

[2] F. Matúš and J. Flusser, "Image representation via a finite Radon transform," IEEE Transactions on Pattern Analysis \& Machine Intelligence, vol. 15, no. 10, pp. 996-1006, Oct 1993.

[3] JP. Guédon and N. Normand, "The Mojette transform: the first ten years," in Proc. 12th International Conference on Discrete Geometry for Computer Imagery, É. Andres, G. Damiand, and P. Lienhardt, Eds., Poitiers, France, Apr 2005, vol. LNCS3429, pp. 79-91, SpringerVerlag.

[4] M. Katz, Questions of uniqueness and resolution in reconstruction from projections, Lect. Notes in Biomath., Springer Verlag, 1977.

[5] N. Normand, JP. Guédon, O. Philippé, and D. Barba, "Controlled redundancy for image coding and high-speed transmission," in Proc. SPIE Visual Communications and Image Processing 1996, R. Ansari and MJ. Smith, Eds. SPIE, Feb 1996, vol. 2727, pp. 1070-81.

[6] N. Normand, A. Kingston, and P. Évenou, "A geometry driven reconstruction algorithm for the Mojette transform," in Proc. 13th International Conference on Discrete Geometry for Computer Imagery, A. Kuba, LG. Nyúl, and K. Palágyi, Eds., Szeged, Hungary, Oct 2006, vol. LNCS4245, pp. 122-33, Springer-Verlag.

[7] M. Servières, J. Idier, N. Normand, and JP. Guédon, "Conjugate gradient Mojette reconstruction," in Proc. SPIE Medical Imaging 2005: Image Processing, JM. Fitzpatrick and JM. Reinhardt, Eds., Apr 2005, vol. 5747, pp. 2067-74.

[8] M. Luby, "Lt codes," in Proc. 43rd Ann. IEEE Symp. Found. Comp. Sci., Nov. 2002, pp. 271-280.

[9] F. Zhai, Y. Eisenberg, and AK. Katsaggelos, Joint sourcechannel coding for video communications, Elsevier Academics Press, 2nd Edition, 2005. 